\title{
Combination of Hybrid Chaotic Encryption and LDPC for Secure Transmission of Images over Wireless Networks
}

\author{
Mona F. M. Mursi \\ Prof. Dept. Of Electrical Engineering, Shubra Faculty of Engineering, Benha University, Egypt. \\ monmursi@yahoo.com, \\ Hossam Eldin H. Ahmed \\ Dept. Of Electronics Comm. Eng., P. Dean of the Faculty of Electronic Eng. Menouf-32952, Menufiya University, \\ Egypt. \\ hhossamkh@yahoo.com \\ Fathi E. Abd El-samie \\ Assoc. Prof. Dept. Of Electronics\& Communication Engineering, Faculty of Electronic Eng - Menufiya University. \\ Egypt. \\ fathi_sayed@yahoo.com,
}

\author{
Ayman H. Abd El-aziem \\ Ph. D Student, Dept. Of Electrical Engineering, Shubra Faculty of Engineering, Benha University, Egypt. \\ eng_aymnhassnin@yahoo.com
}

\begin{abstract}
Robust and secure transmission strategy for high quality image through wireless networks is considered a great challenge. However, the majority of encrypted image transmission schemes don't consider well the effect of bit errors occurring during transmission. These errors are due to the factors that affect the information such as noise and multipath propagation. That should be handled by an efficient channel coding scheme. Our proposed scheme is based on combining hybrid chaotic encryption, which is based on twodimensional chaotic maps which is utilized for data security, with an error correction technique based on the Low Density Parity Check (LDPC) code. The LDPC is employed as channel coding for data communication in order to solve the problem of the channel's limited bandwidth and improve throughput. Simulation results show that the proposed scheme achieves a high degree of robustness against channel impairments and wide varieties of attacks as wells as improved reliability of the wireless channel. In addition, LDPC is utilized for error correction in order to solve the limitations of wireless channels.
\end{abstract}

Index Terms-LDPC, Turbo, Chaotic Ma, Network security, error corrections.

\section{INTRODUCTION}

The wireless communication medium, as opposed to the wired counterparts, is noisy and open to intruders. Hence, an additional level of error protection and security is required to make the wireless network as reliable and secure as the wired network [1]. Error control codes (ECC) are an important issue in wireless transmission, and are used to protect data from channel errors. The issue of using cryptography, secure ciphers in the wireless noisy channel is that the very same property that gives ciphers their cryptographic strength makes them sensitive to channel errors. This sensitivity causes retransmissions thus reducing the overall throughput. To improve the throughput in noisy environments, channel coding is performed after encryption. There are many factors that cause the output of a communication channel to be different from its input. Among these factors are attenuation, nonlinearities, bandwidth limitations, multipath propagation and noise [2]. For reliable communications, to improve the throughput in noisy environments, channel coding is performed after encryption.

There are many researchers who have introduced error correction and encryption in communication networks that have been addressed independently such as in [3-4]. Several researchers have studied the trade-off between encryption and error correction by trying to combine these functionalities in one unit. For instance, Gligoroski et al [5].

In this paper, we introduce a new algorithm which combines encryption and coding in one algorithm; we propose hybrid chaotic encryption with LDPC. The paper will be organized as follows: section 2 gives a brief discussion of channel coding techniques as LDPC and Turbo code technique. Section 3 presents a comparison between the performance of LDPC and Turbo. Section 4 
defines the performance parameters. Section 5 explains the proposed scheme. Experimental results are discussed in section 6. Finally, Section 7 concludes the paper.

\section{Channel Coding}

Channel coding techniques are divided into two main types; block codes and convolution codes. In this section we introduce two different algorithms one is LDPC code as a type of block code, and the other is Turbo code as a type of convolution code [6-7].

\section{A. LDPC Code}

LDPC is an error correcting code used in noisy communication channels to reduce the probability of loss of information. With LDPC, this probability can be reduced as small as desired, thus the data transmission rate can be as close to Shannon's limits [8].

Nowadays, LDPC has made its way into some modern applications such as 10GBase-T Ethernet, Wi-Fi, WiMAX, Digital Video Broadcasting (DVB). Since 1993, with the invention of turbo codes, researchers have switched their focus to finding low complexity codes which can approach Shannon channel capacity [9-10].

If a code uses $n$ bits to provide error protection to $\mathrm{k}$ bits of information, it is called a (n, k) block code. In the case of image transmission, larger $\mathrm{n}$ and $\mathrm{k}$, a matrix representation of the codes is used. This representation includes a generator matrix, $\mathrm{G}$, and a parity-check matrix, $H$. Given a message $p$, the codeword $c$ will be the product of $\mathrm{G}$ and $\mathrm{p}$ with entries modulo 2:

$$
c=p \times G
$$

Given the received codeword y, the syndrome vector is

$$
z=y \times H
$$

If $z=0$ then the received codeword is error-free, else, the value of $\mathrm{z}$ is the position of the flipped bit. For more details about LDPC coding and decoding refer to [9-10].

\section{B. Turbo Channel Coding}

Turbo codes use an interleaver between two parallel or serial encoders as shown in figure 1 the existence of the interleaver results in very large code word lengths with excellent performance, particularly at low SNRs. Using these codes, it is possible to get as close as $0.7 \mathrm{~dB}$ to the Shannon limit at low SNRs. The turbo encoder consists of two constituent codes separated by an interleaver of length $\mathrm{N}$. Because the encoders are systematic, each encoder generates the $\mathrm{N}$-information bits applied to its input followed by $\mathrm{N}$ parity check bits. After the encoding, the $\mathrm{N}$ information bits and the $2 \mathrm{~N}$ parity check bits of the two encoders, a total of $3 \mathrm{~N}$ bits, are transmitted over the channel. Therefore, the overall rate is $\mathrm{R}=\mathrm{N} / 3 \mathrm{~N}=1 / 3$.

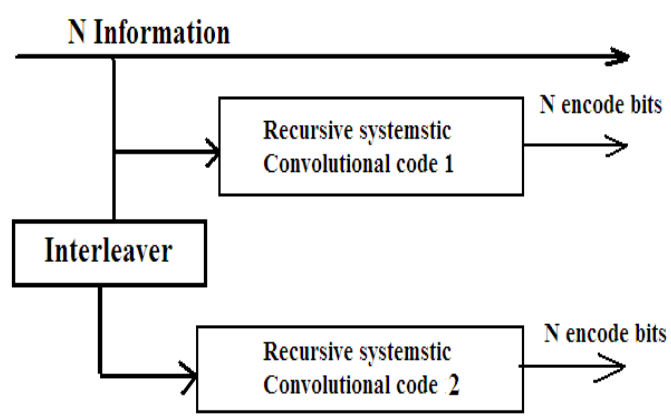

Fig. 1. Block diagram of Turbo encoder

The interleaver in turbo codes is usually very long, of the order of thousands of bits. Pseudorandom interleavers perform well, although some improvement in the performance of the code can be obtained by clever choice of the interleaver. This improvement is more noticeable at short interleaver lengths. Due to the existence of the interleaver, it is, in most cases, impossible to return both codes to the all-zero state.

Since turbo codes have two constituents-code components, an iterative algorithm is appropriate for their decoding. Any decoding method that yields the likelihood of the bits as its output can be used in the iterative decoding scheme as shown in figure 2. One such decoding scheme is the maximum a posteriori (MAP) decoding method.

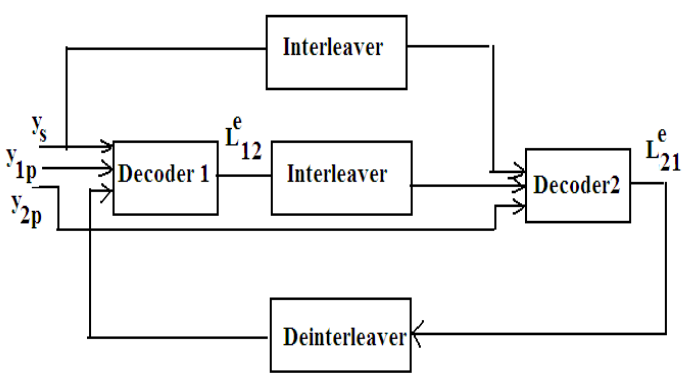

Fig. 2. Block diagram of Turbo decoder

For more details about turbo coding and decoding, refer to [11].

\section{Performance Parameters}

The quality of the received image can be measured by many performance parameters such as the Peak Signal to Noise Ratio (PSNR). It is most commonly used as a measure of the quality of a reconstructed image. It is most easily defined via the Mean-Squared Error (MSE) which for two $\mathrm{m} \times \mathrm{n}$ monochrome images $\mathrm{I}$ and $\mathrm{K}$ where one of the images is considered a noisy approximation of the other. It is defined as:

$$
M S E=\frac{1}{m \times n} \sum_{i=0}^{m-1} \sum_{j=0}^{n-1}\|I(i, j)-K(i, j)\|^{2}
$$


The PSNR is defined as:

$$
P S N R=10 \log _{10}\left(\frac{255^{2}}{M S E}\right)
$$

The other parameters are Message Error Rate (MER) and Bit Error Rate (BER), MER define as = (No. of messages received in Error after error correction) / (Total no. of messages transmitted) [6].

BER quantifies the reliability of the entire radio system it can be defined as: BER = Errors/Total Number of Bits with a strong signal and an unperturbed signal path, this number is too small as to be insignificant. It becomes significant when we wish to maintain a sufficient signalto-noise ratio in the presence of imperfect transmission through electronic circuitry.

\section{COMPARISON BetweEn PERFormance OF Turbo AND LDPC}

The comparison of different channel coding techniques will help to identify the most efficient one for a particular application. After the remarkable work [12] of Claude Shannon in 1948 it is necessary to transmit errorless data mainly in digital communications which are being used for multimedia communications for small hand held devices, such as Mobile Phones, Pagers, PDAs etc.

We compare between the performance of LDPC and turbo. In BER and processing time to determine the best for use in our proposed algorithm, we use the two algorithms with the following parameters.

The time is measured from the time the image inputs to be encoded to the end of the decoding of the image then reconstructing the decoded image.

Table 1.Simulation parameters of comparison between LDPC and Turbo codes

\begin{tabular}{|c|c|c|}
\hline & LDPC & Turbo \\
\hline Modulation & BPSK & BPSK \\
\hline Coding & $\begin{array}{l}\text { Rate-1/2, block } \\
\text { length 512-bit }\end{array}$ & $\begin{array}{l}\text { Rate-1/2, block } \\
\text { length 512-bit }\end{array}$ \\
\hline $\begin{array}{c}\text { No of } \\
\text { Iterations }\end{array}$ & 25 & 2 \\
\hline SNR (dB) & {$[0: 3.7]$} & {$[0: 3.7]$} \\
\hline $\begin{array}{c}\text { Channel } \\
\text { estimation }\end{array}$ & Gaussian channel & Gaussian channel \\
\hline Input image & $\begin{array}{c}\text { Cameraman } \\
256 \times 256\end{array}$ & $\begin{array}{c}\text { Cameraman } \\
256 \times 256\end{array}$ \\
\hline
\end{tabular}

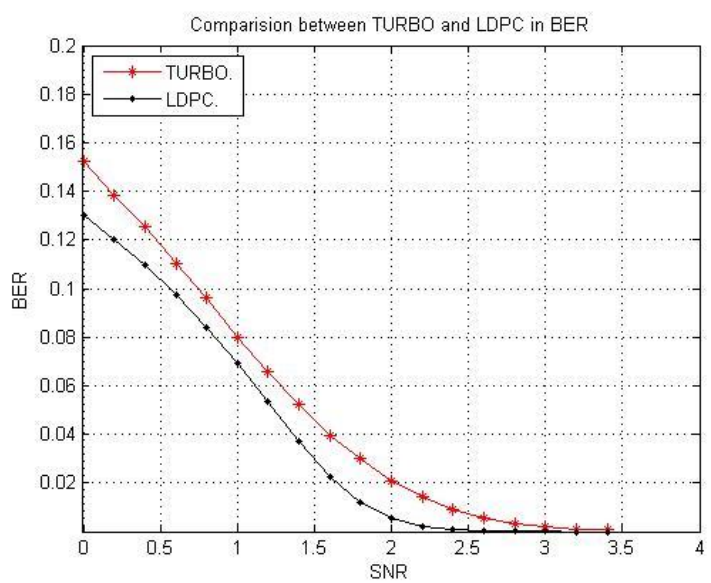

Fig. 3. BER versus SNR of the received cameraman image with Turbo and LDPC

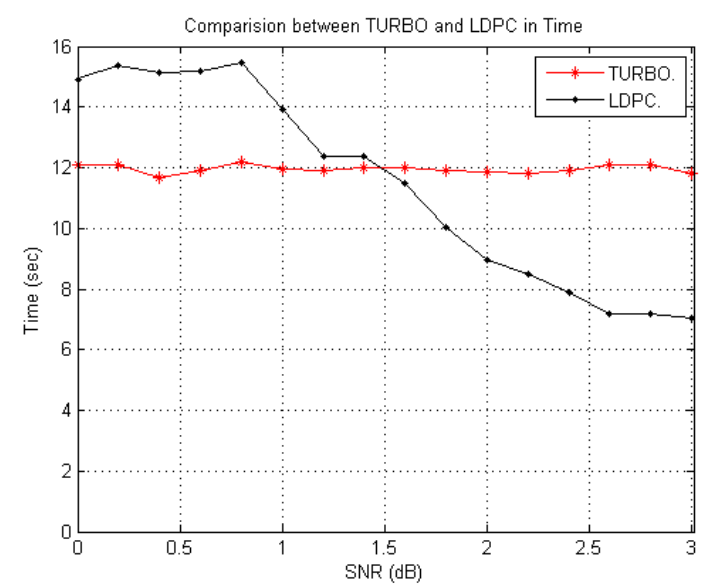

Fig. 4. Time versus SNR of the received cameraman image with Turbo and LDPC

From the previous figure 3 and 4 it is clear LDPC has a small BER than turbo codes and it executes short time with LDPC at SNR from 1.5 to 3 compared to Turbo code so that it preferable to use LDPC as an error correction code in our proposed scheme.

We can improve the performance of turbo code by making some increase number of iterations and decreasing block length that will improve the performance, where these conditions executed long time and it is not suitable for real time application in an image as can be shown in the figures 5 and 6 .

We study the effect of the number of iterations by taking SNR to be changed from 0.2 to 1.5 as sample of noise, and testing the BER and the execution time for correcting the errors. It is clear the performance of turbo code depends mainly on the number of iterations and does not depend on the value of the SNR. 


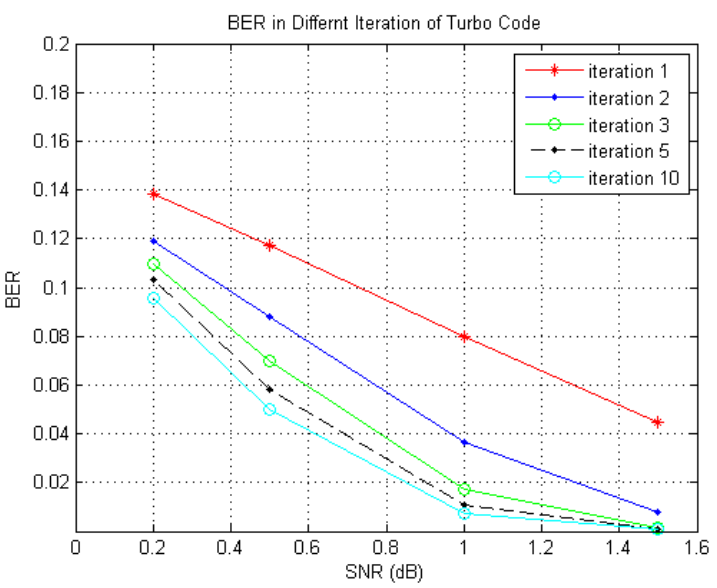

Fig. 5. SNR versus BER of the received Cameraman image with different iteration in Turbo codes

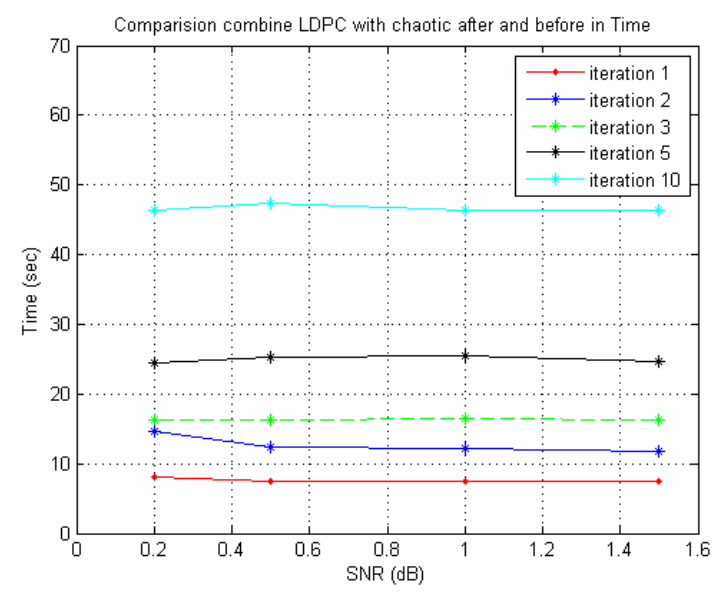

Fig. 6. Processing time at different iteration in Turbo codes

\section{PRocedure OF COMBINING ENCRYPTION WiTH CODING}

The encryption and LDPC algorithms are applied in two different methods; first we applied coding, then encryption as follows: we first applied encoding the original image (cameraman.bmp) using LDPC then applied encryption using the Arnold cat map as the type of encryption [17], we use BPSK for modulation and AWGN as a channel. The second method is using the same algorithm but in this case we applied the encryption first, then coding. At the destination, we applied decode, then decrypted the decoded image. We compare between the two methods by measuring BER, FER, PSNR and processing time. The simulation results of the two methods can be shown in the following figures7, 8, 9 and 10 .

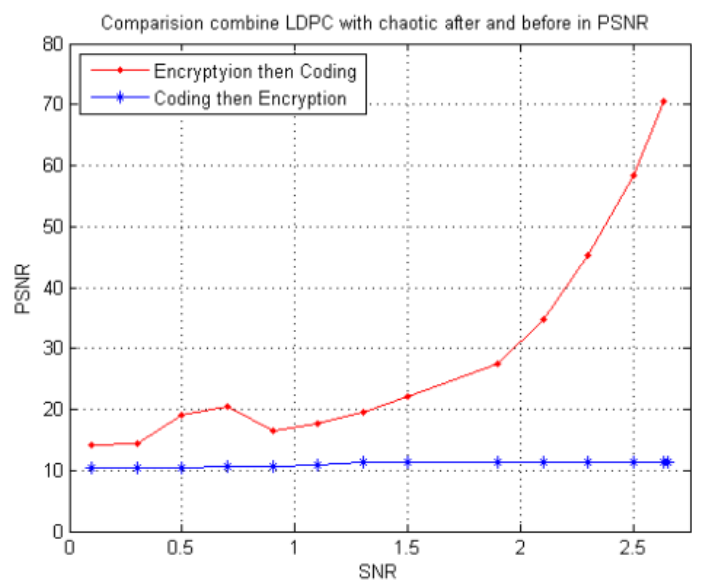

Fig. 7. PSNR for combining encryption with LDPC coding after and before for various SNRs (dB).

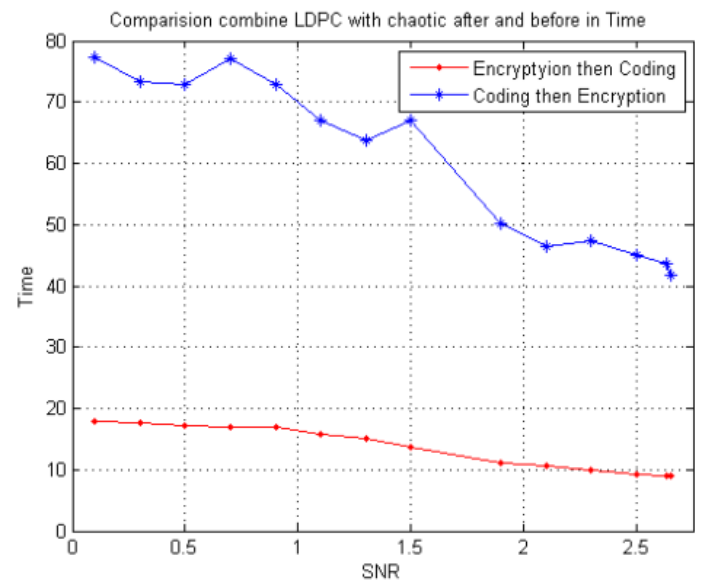

Fig. 8. Processing time for combining encryption with LDPC coding after and before for various SNRs (dB).

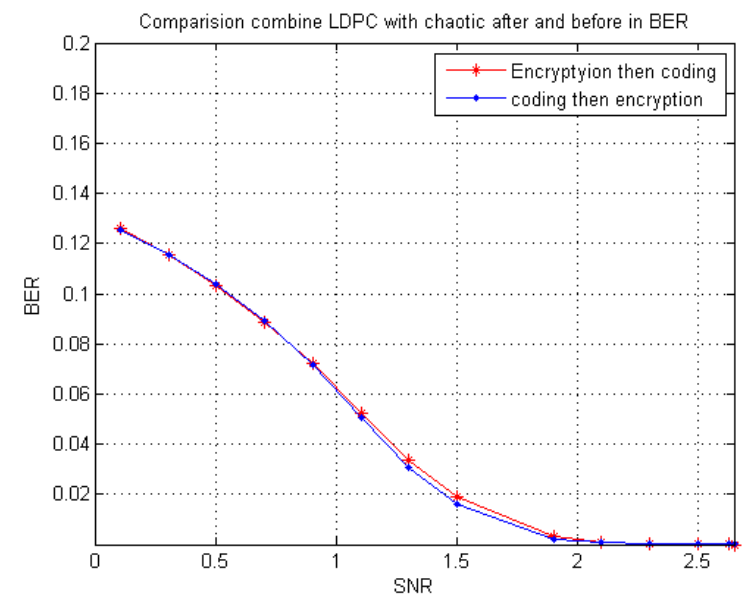

Fig 9. BER for combining encryption with LDPC coding after and before 
From the previous figures $7,8,9$ and 10 we can conclude that:

1- By measuring the parameter PSNR, as can be shown in figure 7 , it is evident that applying encryption, then coding gives better results in the reconstruction of the decoded decrypted image.

2- There are small improvements in BER and FER by applying coding then encryption, as is clear from figures 8 and 9 .

3- The performance of applying encryption then coding has smaller execution time compared to the time executed by applying coding then encryption. That is due to the fact that in the coding then encryption, we encrypt the parity which is added to the image in encoding the image. So combining encryption with coding is preferable to apply encryption and then coding.

\section{COMBINATION OF HYBRID CHAOTIC ENCRYPTION AND LDPC CODE}

We proposed a new schema which combines encryption and then coding in one algorithm. We use a hybrid chaotic system as follow: first an image is transformed to the fractional Fourier transform domain [14]. Secondly, the transformed image is encrypted using two stage encryption scheme of confusion and diffusion presented by Arnold Cat map for confusion and proposed Hénon map3 for diffusion [14]. Finally the encrypted image is obtained. More details about this algorithm are shown in [14]. We combine this encryption algorithm with LDPC codes for channel coding as shown of flowchart of figure 11. The encoded encrypted image is then modulated by Binary Phase Shift Keying (BPSK), transmission over channel Additive white Gaussian noise (AWGN), which is considered in our simulation. AWGN is a basic noise model used in information theory to mimic the effect of many random processes that occur in nature. At the receiver side, the received image was demodulated, decoded and decrypted. After decryption, we will pass the image through a median filtering to reduce noise.

We compare our proposed algorithm by combining LDPC code with three different algorithms. First applying RC6 [15], second applying Baker chaotic map [16], and finally applying LDPC without encryption. We compare among all these algorithms in tests for BER, FER, and PSNR. We will carry out a comparison between the image transmission using this proposed scheme and the transmission of the original image using the previous algorithms. We compare between them to determine the performance of our proposed scheme and measure the ability for secure image transmission and error correction in the noisy channel.

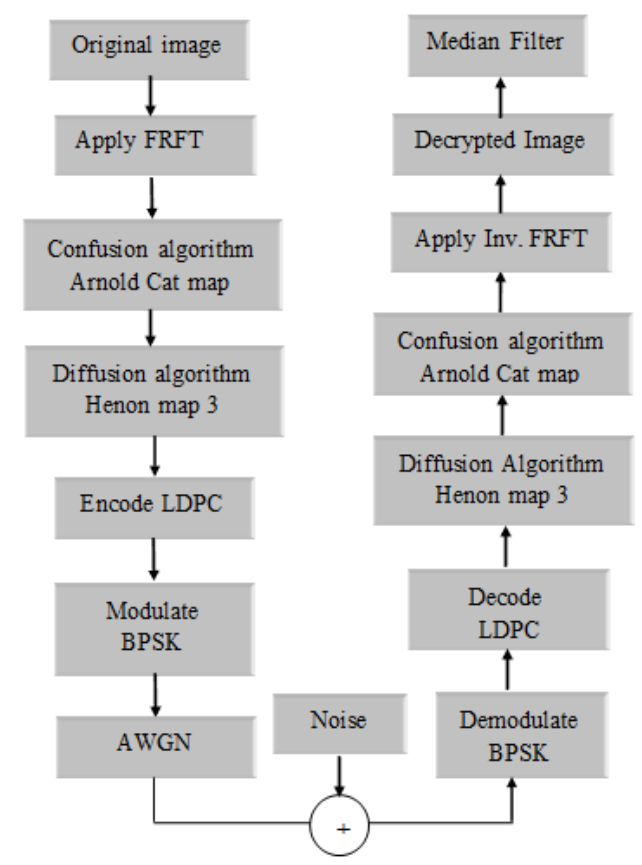

Fig. 11. Combination of hybrid chaotic map with LDPC code in a Shannon channel

\section{EXPEREMENTAL RESULTS}

The performance of the combination between hybrid chaotic map and LDPC channel coding for image transmission through AWGN channel has been demonstrated under the simulation parameters shown in Table 2.

Table 2 Simulation parameters of proposed scheme

\begin{tabular}{|l|c|}
\hline Modulation & BPSK modulation \\
\hline Coding & $\begin{array}{c}\text { LDPC coding } \\
\text { Code rate }=1 / 2 \\
\text { Block length } 512 \text {-bit } \\
\text { No of Iterations }=25\end{array}$ \\
\hline SNR (dB) & From 0 to 2.75 \\
\hline Channel & AWGN channel \\
\hline Filter & Median filter \\
\hline Ciphering & Hybrid chaotic encryption using FRFT \\
\hline
\end{tabular}

We combine three different image encryption algorithms with LDPC to decide on the best of them. First we combine Baker chaotic map with LDPC code [16]. Second, combine RC6 with LDPC code [15]. Third apply LDPC without encryption; finally our proposed algorithm hybrid chaotic map using FRFT [14] combined with LDPC code. We make comparisons among all these algorithms in some tests as BER, FER, and PSNR. The result of the combination of different encryption and LDPC can be shown in the following tables and figures. 
Table 3 PSNR for different encryption models combine with LDPC

\begin{tabular}{|c|c|c|c|c|}
\hline SNR & $\begin{array}{c}\text { Without } \\
\text { encryption }\end{array}$ & Chaotic & RC6 & $\begin{array}{c}\text { Proposed hybrid } \\
\text { chaotic map } \\
\text { using FRFT }\end{array}$ \\
\hline 0 & 13.92 & 13.56 & 13.55 & 13.57 \\
\hline 0.5 & 14.84 & 14.56 & 14.58 & 14.57 \\
\hline 1 & 17.11 & 16.87 & 16.90 & 16.89 \\
\hline 1.5 & 23.00 & 23.19 & 22.83 & 23.00 \\
\hline 2 & 34.72 & 34.99 & 34.70 & 35.54 \\
\hline 2.5 & 49.94 & 52.96 & 53.91 & 65.26 \\
\hline 2.75 & $\infty$ & 72.47 & 72.42 & 72.42 \\
\hline
\end{tabular}

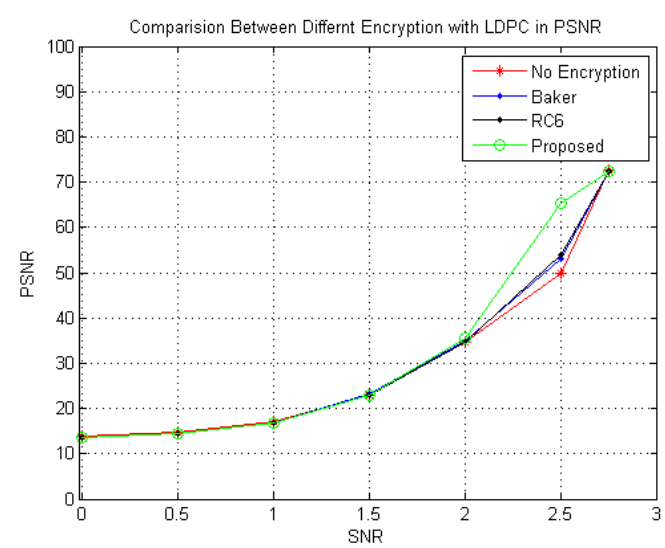

Fig. 12. PSNR vs. SNR for combining different encryption models with LDPC

\section{A. Peak Signal to Noise Ratio}

Table 3 and figure 12 show the PSNR against BER. Which are the measurements of the ratio between the maximum possible power of a signal and the power of corrupting noise that affects the fidelity of its representation. PSNR values are measured Appling four models for the combination of Hybrid chaotic and LDPC coding for various Signal to Noise Ratios (SNRs) of the received cameraman image. The higher PSNR for the proposed scheme indicates more efficient system performance.

Table 4 FER for different encryption models with LDPC

\begin{tabular}{|c|c|c|c|c|}
\hline SNR & $\begin{array}{c}\text { Without } \\
\text { encryption }\end{array}$ & Chaotic & RC6 & $\begin{array}{c}\text { Proposed } \\
\text { hybrid chaotic } \\
\text { map using } \\
\text { FRFT }\end{array}$ \\
\hline 0 & 1 & 1 & 0.9997 & 0.9997 \\
\hline 0.5 & 0.9877 & 0.9856 & 0.9814 & 0.9824 \\
\hline 1 & 0.7417 & 0.7434 & 0.7385 & 0.7441 \\
\hline 1.5 & 0.2263 & 0.2089 & 0.2270 & 0.2187 \\
\hline 2 & 0.02002 & 0.0168 & 0.0166 & 0.0151 \\
\hline 2.5 & 0.00073 & 0.00024 & 0.00048 & 0.00024 \\
\hline 2.75 & 0 & 0 & 0.00024 & 0 \\
\hline
\end{tabular}

Table 5 BER for different encryption models combine with LDPC

\begin{tabular}{|c|c|c|c|c|}
\hline SNR & $\begin{array}{c}\text { Without } \\
\text { encryption }\end{array}$ & Chaotic & RC6 & $\begin{array}{c}\text { Proposed hybrid } \\
\text { chaotic map } \\
\text { using FRFT }\end{array}$ \\
\hline 0 & 0.13069 & 0.1304 & 0.13035 & 0.13015 \\
\hline 0.5 & 0.10399 & 0.1037 & 0.10338 & 0.10346 \\
\hline 1 & 0.06082 & 0.0606 & 0.060712 & 0.060706 \\
\hline 1.5 & 0.01555 & 0.0142 & 0.015591 & 0.014794 \\
\hline 2 & 0.00109 & 0.00097 & 0.000993 & 0.000866 \\
\hline 2.5 & $3.29 \mathrm{e}-5$ & $1.52 \mathrm{e}-5$ & $1.57 \mathrm{e}-5$ & $9.53 \mathrm{e}-7$ \\
\hline 2.75 & 0 & 0 & 0 & 0 \\
\hline
\end{tabular}

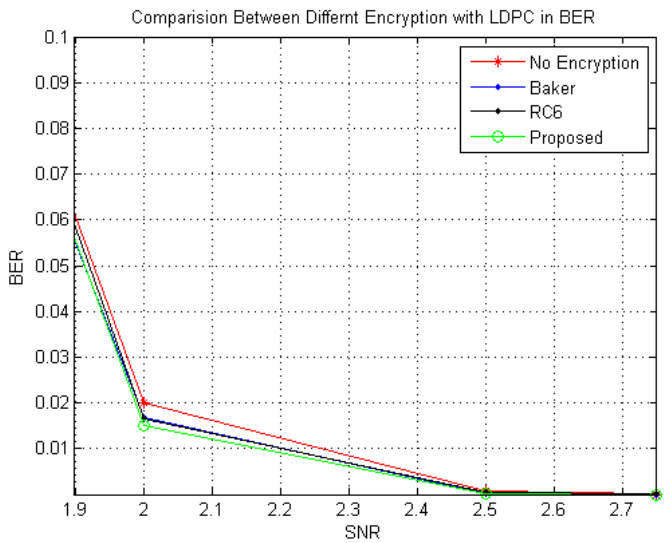

Fig. 13. BER for combining different encryption models with LDPC

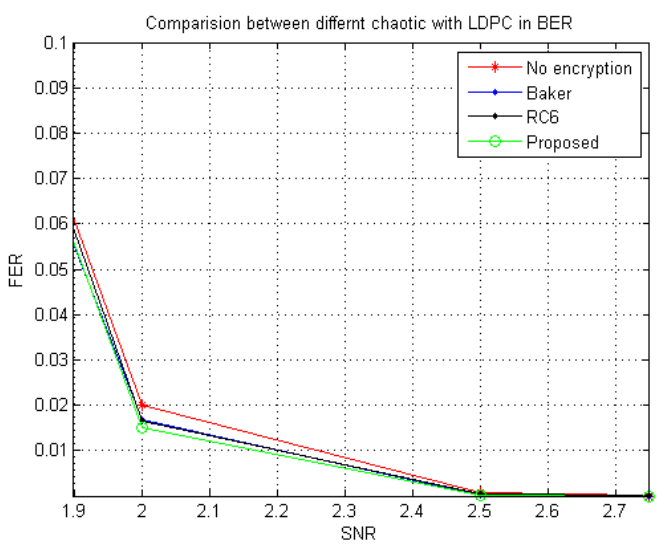

Fig. 14. FER for combining different encryption models with LDPC

\section{B. Bit Error Rate and Frame Error Rate}

Tables 4, 5 and figures 13 and 14 illustrate the BER and FER versus SNR respectively. BER and FER are computed after decoding, as a function of signal to noise ratio. BER and FER are measured for four models of combination of Hybrid chaotic and LDPC code for various SNRs. It is clear that the proposed scheme has very a small value of the BER and FER at small SNR so that it has better error performance.

From the previous figures and tables it is clear that our proposed algorithm with LDPC gives the best results compared with RC6, Baker and the original image without encryption. 


\section{Histogram Analysis}

The original image can be shown in figure 15, the histogram of the original image and its encrypted image using a hybrid chaotic map is given in Figure 16 and 17. These figures show that the histogram of the encrypted image is fairly uniform and completely different from that of the original image.

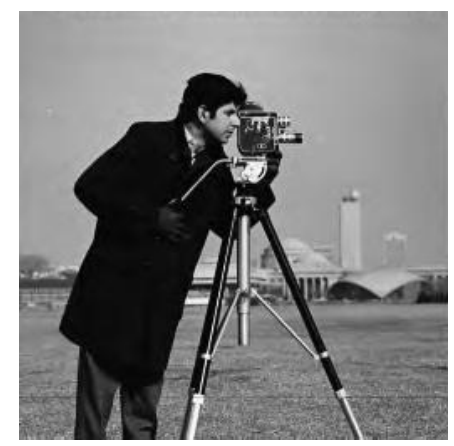

Fig. 15. Original Cameraman Image

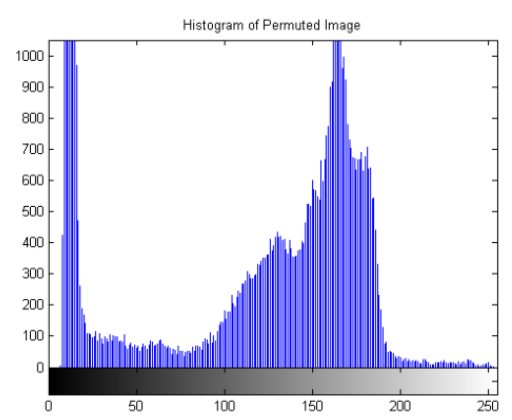

Fig. 16. histogram of original image

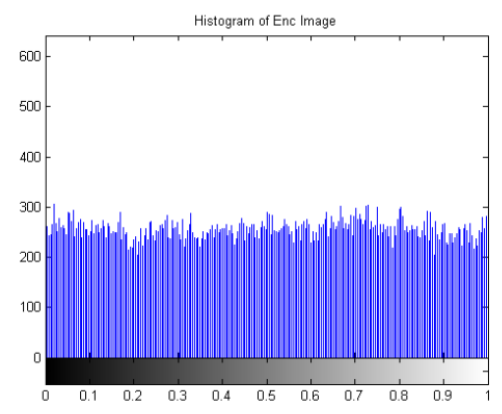

Fig. 17. Histogram of encrypted image

We introduce the performance analysis of our proposed algorithm to secure transmission of image through a noisy channel by drawing the following three figures, which measure the BER FER and processing time. It is clear that our proposed algorithm gives very small BER at small SNR and it has also high PSNR at small SNR. It executes at a short time when applying encryption, encoding, decoding and decryption of image all these algorithms execute in $14.55 \mathrm{sec}$ at $\mathrm{SNR}=2$. Also, it can reconstruct the decoded decrypted image as the original image with noisy channel, besides, it could secure the transmission image as it clear from the histogram of the encrypted image in figure 17.

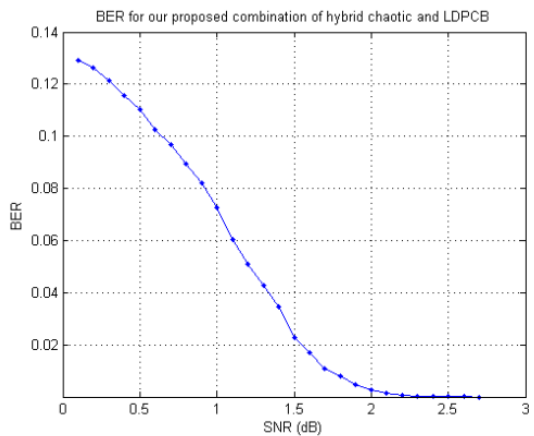

Fig. 18. BER vs. SNR of the received image combination of hybrid chaotic encryption and LDPC

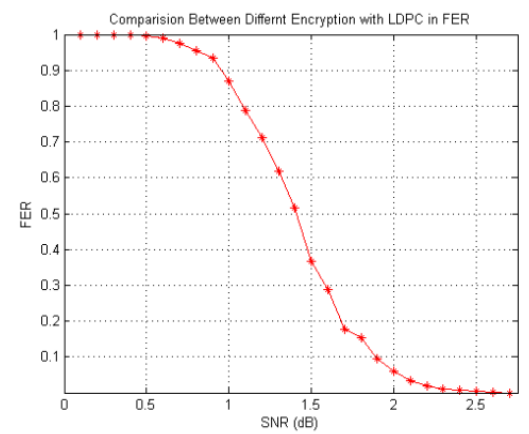

Fig. 19. FER vs. SNR of the received Cameraman image with combination hybrid chaotic and LDPC

The following figures are produced from applying our proposed algorithm to secure transmission of the image besides error correction. We show the ability of the algorithm to correct the errors at different SNRs

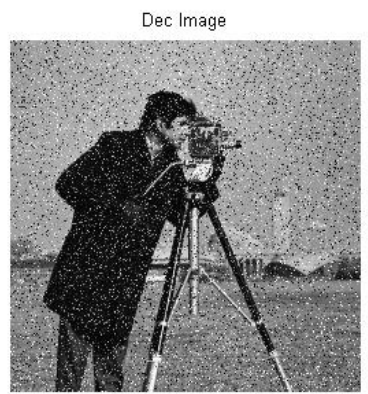

Fig. 20.Reconstructing image at $S N R=1 d B$

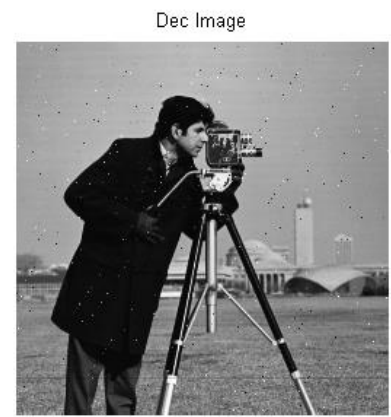

Fig. 21. Reconstructing image at $S N R=2 d B$ 


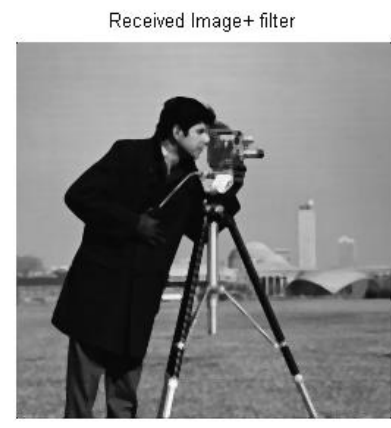

Fig. 22. Reconstructing image at $\mathrm{SNR}=2 \mathrm{~dB}$ using median filter

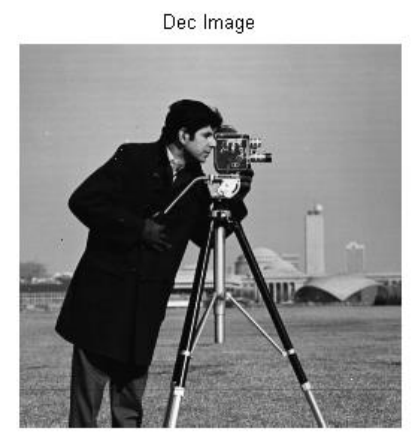

Fig. 23. Reconstructing image at $\mathrm{SNR}=2.5 \mathrm{~dB}$ image besides error correction

Figures 20, 21, 22 and 23 give the reconstructed image of the proposed scheme at different values of SNR over AWGN channels. As shown a small change in SNR yields high quality in the reconstructed image.

It is observed that in the case of the reconstructed image for a channel with $\mathrm{SNR}=1,2,2.5 \mathrm{~dB}$ with the combination of hybrid chaotic map and LDPC coding, it is possible to reconstruct the image clearly with the combination of LDPC coding, and the image is reconstructed without distortion. we don't need to retransmission of image when it noisy therefore our proposed algorithm increases the throughput of the system. The use of median filter on the receiver side improves the reconstruction of the image in the presence of noise as is clear in figure 22.Our proposed scheme is effective for secure image communication over the wireless noisy channel.

\section{CONCLUSION}

In this paper, we proposed a scheme for the combination of image encryption and error correction code. The proposed scheme combines image encryption based on hybrid chaotic encryption, and error control coding based on LDPC channel coding. Simulation results show that the proposed scheme enhanced the performance parameters and achieved both security and reliability of image transmission through the wireless channel. The proposed scheme is suggested for secure image transmission over wireless channels. It proposes to apply encryption before coding because of shorter execution time than when applying coding, prior to encryption; besides, it gives good result in the reconstruction of the image at the receiver side. Also as is evident from the results in this paper, LDPC gives better results than Turbo code. Our proposed scheme yields better results than RC6, Baker map and without encryption. Finally we can conclude that our proposed scheme is very effective for secure image communication over wireless noisy channels. Further works is needed for developing an optimization scheme for the security issues and error correction code for wireless image transmission.

\section{REFERENCES}

[1] M. A. El-Iskandarani, Saad M. Darwish, Saad M. Abuguba, Combination of 2D chaotic Encryption and Turbo Coding for Secure Image Transmission, IJCSNS International Journal of Computer Science and Network Security, VOL.10 No.11, November 2010.

[2] Daniel J. Costello, Jr. and G. David Forney, Jr, Channel Coding: The Road to Channel Capacity, IEEE, Vol. 95, No. 6, June 2007.

[3] Deergha Rao"A Robust and Secure Scheme for Image Communication over Wireless Channels", IEEE CAS on ETW Conference, pp.88-91, 2005.

[4] A. Padmaja and M. Shameem, "Secure Image Transmission over Wireless Channels", IEEE ICCIMA Conference, Sivakasi, Tamil Nadu, pp.44-48, January 2007.

[5] D. Gligoroski, S. Knapskog, and S. Andova, "Cryptcoding - Encryption and Error Correction Coding in a Single Step" International Conference on Security and Management, pp. 1-7, June 2006

[6] Mona F. M. Mursi, Hossam Eldin H. Ahmed, Fathi E. Abd El-samie, Ayman H. Abd El-aziem" Image Security With Different Techniques Of Cryptography And Coding: A Survey" IOSR Journal of Computer Engineering (IOSR-JCE) e-ISSN: 2278-0661,Volume 16, Issue 3, Ver. I (May-Jun. 2014), PP 39-45.

[7] Mr. Vishal G. Jadhao, Prof. Prafulla D. Gawande, Performance Analysis of Linear Block Code, Convolution code and Concatenated code to Study Their Comparative Effectiveness, IOSR Journal of Electrical and Electronics Engineering (IOSRJEEE), SSN: 2278-1676 Volume 1, Issue 1 (May-June 2012), PP 53-61.

[8] Jorge C. Moreira and Patrick G. Farrell "Essential of Error Control Coding," John Wiley \& Sons Ltd, The Atrium, Southern Gate, Chichester, West Sussex PO19 8SQ, England. 2006.

[9] A Tutorial on Low Density Parity-Check Codes, Tuan Ta, The University of Texas at Austin, Internet resources.

[10] Bernhard M. J. Leiner, "LDPC Codes- a brief Tutorial", April 8, 2005.

[11] C.Berrou and A. Glavieux,"Near Optimum Error Correcting Coding and Decoding: Turbo Codes" IEEE transactions on communications Vol.44, No: 10, pp.12611271, 1996.

[12] C. Shannon, "A Mathematical Theory of Communication", Bell System Technical Journal, Vol. 27, pp. 379-423, 1948.

[13] Arpita Ray Sarkar, Subhankar Majhi "Comparison of Channel Coding Techniques Reference to Image Storage 
and Retrieval" International Journal of Engineering and Innovative Technology (IJEIT) Volume 1, Issue 5, 2012.

[14] Mona F. M. Mursi, Hossam Eldin H. Ahmed, Ayman H. Abd El-aziem "Image Encryption Based On Development of Hénon Chaotic Maps Using Fractional Fourier Transform" Accepted for publish at (MCSI 2014) Varna, Bulgaria, September 13-15, 2014

[15] H. E. H. Ahmed, H. M. Kalash, and O. S. Faragallah, "Encryption Efficiency Analysis and Security Evaluation of RC6 Block Cipher for Digital Images", International Conference on Electrical Engineering, pp. 1-7, April 2007.

[16] J. Fridrich. Image encryption based on chaotic ma.ps. In Proc. IEEE Int. Conference on systems, Man and Cybernet-ics, volume 2, pages 1105-1110, 1997.

[17] Zhu Liehuang, Li Wenzhuo, Liao Lejian, Li Hong. 2006. A Novel Algorithm for Scrambling Digital Image Based on Cat Chaotic Mapping. Proceedings of the 2006 International Conference on Intelligent Information Hiding and Multimedia Signal Processing (IIH-MSP'06), 0-7695-2745-0/06 @ IEEE.

Prof. Mona F. M. Mursi received the B.S.E.E. from Faculty of Engineering, Cairo University, Egypt in 1965, M.S.E.E University of Missouri, Columbia, Missouri, U.S.A. in 1969 and Ph.D. (Computers/ Electronics Engineering) from University of Missouri, Columbia, Missouri, U.S.A. in 1972. She has professional experience at the following: More than 10 years of technical management and professional experience in small to large scale computer systems, peripherals and instrumentation, extensive teaching experience (more than 25 years) of postgraduate and undergraduate courses in computers and electronics / communications, ten years department head experience at EE and Computer Department of Faculty of Engineering and the College of Computers and Information Science, member of several high level academic committees, extensive consultation experience. Her current researches interested are arabization of computer systems, pattern recognition, computer security, biomedical applications of neural networks, microprocessor-based controllers.
Prof. Hossam El din H. Ahmed received the BSC (Honor), MSc, degree from Faculty of Engineering Alexandria University in 1969 and 1977 respectively and a PhD in June 1983(High Institutes of electronics and optics Paul Sabatier University Toulouse France). In 1993 He become a professor of microelectronics, VLSI design technology, communication systems, computer Networks. From 1993 to 1999 he was vice dean for education and student affairs at the faculty of electronics engineering. At 2002 he became the dean of faculty electronics engineering. His current research interested are electrons and ion beams into amorphous polycrystalline target; digital optics; multimedia communication; digital image, communication security application, satellite communication.

Fathi E. Abd El-samie received the BSC (Honor), MSc, and $\mathrm{PhD}$ degree from Menofia university, Egypt, in 1998, 2001, 2005, respectively. He has been the teaching staff member with the department of electronics and electrical communication, faculty of electronic engineering. He is currently a research at KACST - TIC in radio frequency and photonics for the esociety. $\mathrm{He}$ is co- other about 200 paper in international conferences proceeding and journal and four textbooks, his current research interests at image enhancement, image interpolation, super resolution, data hiding, reconstruction of image, multimedia communication.

Ayman H. Abd Alaziem graduated from military technical college (MTC), Egypt in 2002, received his Msc in electric engineering technologies from faculty of engineering Benha University. $\mathrm{He}$ is $\mathrm{PhD}$ candidate in the Department electric engineering at Shubra faculty of engineering Benha University of. His areas of interest are: Chaos theory, Chaos encryption schemes, channel communications and image processing.

How to cite this paper: Mona F. M. Mursi, Hossam Eldin H. Ahmed, Fathi E. Abd El-samie, Ayman H. Abd Elaziem,"Combination of Hybrid Chaotic Encryption and LDPC for Secure Transmission of Images over Wireless Networks", IJIGSP, vol.6, no.12, pp.8-16, 2014.DOI: 10.5815/ijigsp.2014.12.02 